\title{
Design and Implementation of Mobile Learning using 3G Technology
}

\author{
T.V.SARANYA ${ }^{1}$, Y. N. SUPRAJA ${ }^{2}$, M. FIRDOUSE ALI KHAN ${ }^{3}$ \\ ${ }^{I}$ M.TECH (E.S.), Dept. of E.C.E, Aurora's Technological \& Research Institute, \\ ${ }^{2}$ Assoc. Professor, Dept. of E.C.E, Aurora's Technological \& Research Institute, \\ ${ }^{3}$ Sr.Asst. Professor, Dept. of E.C.E. Aurora's Technological \& Research Institute,
}

\begin{abstract}
Mobile learning is new. It is currently difficult to define, conceptualize and discuss. It could perhaps be a wholly new and distinct educational format, needing to set its own standards and expectations [3]. Mobile learning has becomes another very important complement to the traditional ways of learning after digital learning. The development of the third generation mobile communication technology $3 G$ provides a more adequate technical basis for mobile learning [1]. With the continuous development and improvement of the Internet, mobile Education Network and Mobile Telecommunications' Equipment and the gradual increase in the level of the rural economy and the concept of modern learning lifelong learning' in-depth impact and teachers' requirements for further educational technology, mobile learning becomes tools for teachers to develop and improve their educational technology [2]. With the developing trends in mobile communication technology, transmission of real time audio and videos takes a giant leap. It is more than e-learning in which we only view already stored data. The goal of this innovative project is to create flexible teaching solutions, which will enable the accessing of information with mobile devices for educational activities, and to support learning in a variety of situations.
\end{abstract}

\section{Introduction}

Mobile learning through the use of wireless mobile technology allows anyone to access information and learning materials from anywhere and at anytime. As a result, learners have control of when they want to learn and from which location they want to learn. Also, all humans have the right to access learning materials and information to improve their quality of life regardless of where they live, their status, and their culture. Mobile learning, through the use of mobile technology, will allow citizens of the world to access learning materials and information from anywhere and at anytime. Learners will not have to wait for a certain time to learn or go to a certain place to learn. With mobile learning, learners will be empowered since they can learn whenever and wherever they want. Also, learners do not have to learn what is prescribed to them. They can use the wireless mobile technology for formal and informal learning where they can access additional and personalized learning materials from the Internet or from the host organization. Teachers can use the mobile technology to access training materials and information when they need it for just-in-time training. Just-in-time learning encourages high level learning since learner's access and applies the information right away rather than learns the information and then applies the information at a later time. Trainers are empowered since they can use the mobile technology to communicate with learners from anywhere and at anytime. At the same time, Trainers can access learning resources from anytime and anywhere to plan and deliver their lessons [1].

According to MoLeNet, mobile learning can be broadly defined as 'the exploitation of ubiquitous handheld technologies, together with wireless and mobile phone networks, to facilitate, support, enhance and extend the reach of teaching and learning.. Mobile learning involves connectivity for downloading, uploading and/or online working via wireless networks, mobile phone networks or both, and linking to institutional systems. O' Malley etal Believe that mobile learning refers to the position when the learner is not fixed, not in a pre-specified location and the use of mobile technology to provide learning opportunities carried out by any series of learning.

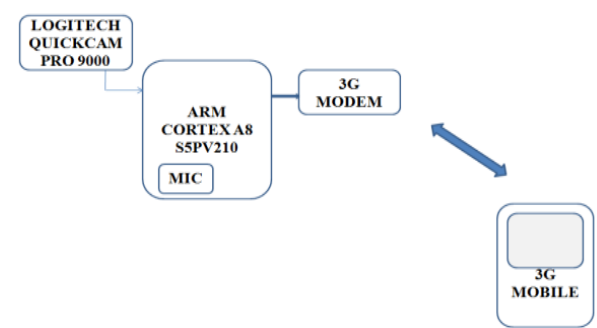

Figure1: Mobile Learning Using 3G Technology 
Mobile learning is not only the use of mobile technology to support learning, to the excessive emphasis on technology rather than to emphasize teaching and learning tasks. Mobile learning is not intended to discover a new kind of mobile technology use, but the emphasis on the teaching and learning process. Mobile learning is the content of learners use the possession of any equipment and technology, in any place, any time, can have the opportunity to enrich their learning.

Although personal computers, laptops, interactive whiteboards, and projection devices have been shown to benefit instructional tasks, their design, cost, usability, and mobility are nevertheless problematic. The size, ease of use, portability, prevalence, and advanced features of mobile technologies (e.g., voice, display, Internet access, interactivity) have sparked interest in integrating these technologies into instructional environments. However, the educational applications of m-technologies are still poorly understood, as is what constitutes good m-learning [4]. "The value of deploying mobile technologies in the service of learning and teaching seems to be both self-evident and unavoidable ", [6]

\section{Tools Used}

SIP: SIP is an application-layer control protocol that can establish, modify, and terminate multimedia sessions (conferences) such as Internet telephony calls. SIP can also invite participants to already existing sessions, such as multicast conferences. Normally SIP uses UDP and TCP port 5060 and TCP 5061 for SSL communication. SIP protocol is very similar to HTTP, so if you have some knowledge about HTTP, then it is easy to learn SIP. SIP doesn't transfer session data like audio, video. RTP (real time protocol) is used for that, SIP just helps to open RTP streams.

\section{Basic SIP Commands}

- $\quad$ INVITE - Initiates a session. This method includes information about the calling and called users and the type of media that is to be exchanged.

- $\quad$ ACK - Sent by the client who sends the INVITE. ACK is sent to confirm that the session is established. Media can then be exchanged.

- $\quad$ BYE - Terminates a session. This method can be sent by either user.

- CANCEL - Terminates a pending request, such as an outstanding INVITE. After a session is established, a BYE method needs to be used to terminate the session.

- OPTIONS - Queries the capabilities of the server or other devices. It can be used to check media capabilities before issuing an INVITE.

- $\quad$ REGISTER - Used by a client to login and register its address with a SIP registrar server.

SIP makes use of transactions to control the exchanges between participants and deliver messages reliably. The transactions maintain an internal state and make use of timers. Client Transactions send requests and Server Transactions respond to those requests with one-or-more responses. The responses may include zero-or-more Provisional (1xx) responses and one-or-more final (2xx-6xx) responses.

Transactions are further categorized as either Invite or Non-Invite. Invite transactions differ in that they can establish a long-running conversation, referred to as a Dialog in SIP, and so include an acknowledgment (ACK) of any non-failing final response (e.g. 200 OK). Because of these transactional mechanisms, SIP can make use of un-reliable transports such as User Datagram Protocol (UDP).

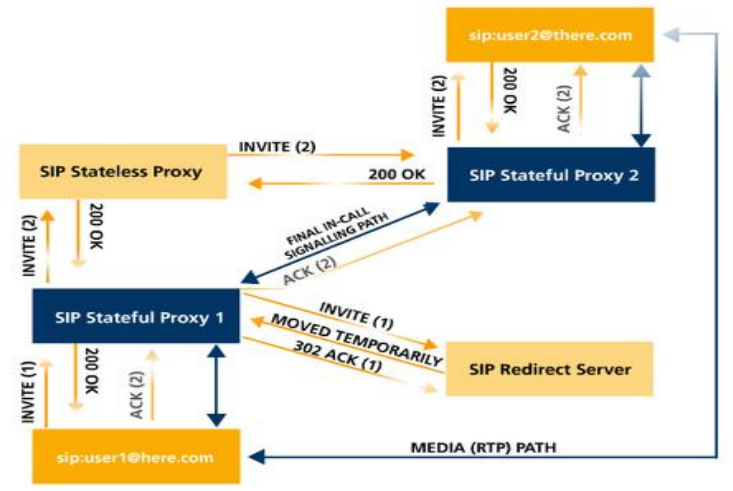

Figure 2: SIP making use of transaction

If we take the above example, User1's UAC uses an Invite Client Transaction to send the initial INVITE (1) message. If no response is received after a timer controlled wait period the UAC may have chosen to terminate the transaction or retransmit the INVITE. However, once a response was received, User1 was 
confident the INVITE was delivered reliably. User1's UAC then must acknowledge the response. On delivery of the ACK (2) both sides of the transaction are complete. And in this case, a Dialog may have been established.

To implement this paper we used oSIP which is an LGPL implementation of SIP. It's stable, portable and flexible The GNU oSIP library is written in C and gets no dependencies except the standard C library. oSIP is thread safe and will generally be used in a multi-threaded application. Nevertheless, this is optional. oSIP is little in size and code and thus could be use to implement IP soft-phone as well as embedded SIP software. oSIP is not limited to endpoint agents, and can also be used to implement "SIP proxy". oSIP does not intend to provide a high layer API for controlling "SIP Session" at this step. Instead, it currently provides an API for the SIP message parser, SDP message parser, and library to handle "SIP transactions".

Linphone: linphone is a SIP web phone with support for several different codec's.

UBUNTU: Ubuntu is an open source OS. In this paper implementation we used ubuntu12.04.

\section{Experimental Results}

Make the connections as per block diagram.

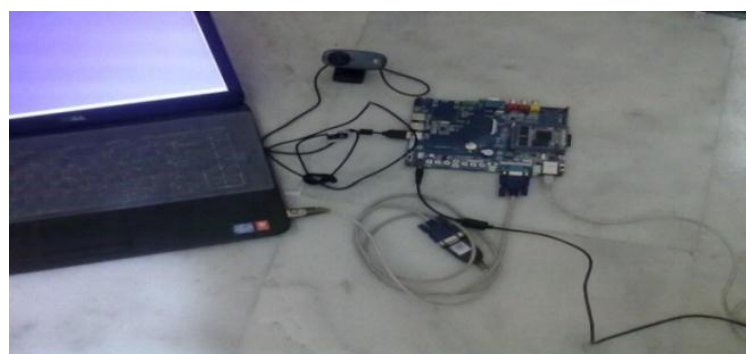

Figure.3: Connecting ARM Board to Laptop for Booting

Note that here laptop is used to boot the ARM board (it is connected to ARM board via RS232 to USB converter cable). After making connections type the commands. This helps in connecting to mobile phone via linphone. Open the terminal of UBUNTU and type the required commands to initiate Linphone then a window will appear as shown in figure 4 .

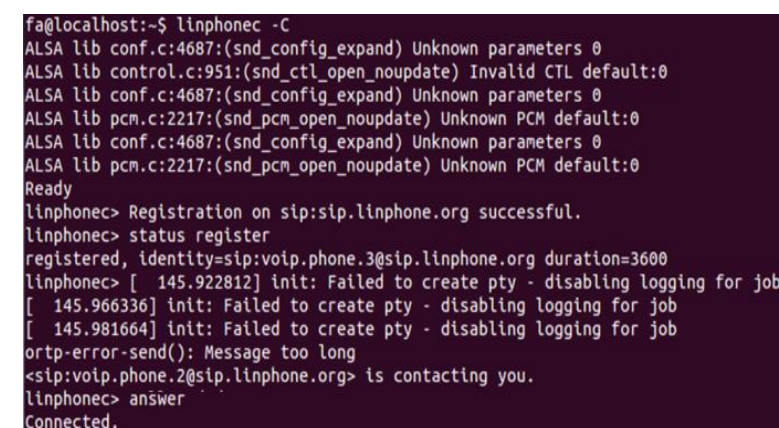

Figure 4: Snap shot showing the establishment of communication using linphone

From mobile phone, using linphone applications call to ARM board. In this case you will get a message on window as shown in figure 4 . Answer it to start conversation from board to mobile phone and we can see the live video of classroom as shown in figure 5 .

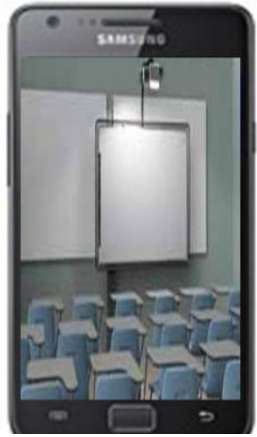

Figure 5: A live video of classroom on $3 \mathrm{G}$ mobile phone 


\section{Advantages}

Mobile learning, through the use of mobile technology, will allow citizens of the world to access learning materials and information from anywhere and at anytime. Learners will not have to wait for a certain time to learn or go to a certain place to learn. With mobile learning, learners will be empowered since they can learn whenever and wherever they want. Teachers can use the mobile technology for just-in-time training where learner's access and applies the information right away rather than learns the information and then applies the information at a later time.

\section{Conclusions}

Mobile technologies are developing rapidly, and the functionalities available on mobile devices grow more numerous and complex every day. These technological advancements, coupled with the widespread availability and relatively low cost of mobile devices, represent a tremendous opportunity to leverage the power and ubiquity of mobile technologies to enhance learning and extend educational opportunities. In 3G systems, it can be realized between the mobile end-user data, audio, video, multimedia communications, which makes anytime, anywhere "easy face to face" communication is possible. Students who are not in classroom can learn the teachers' lectures, as long as opening mobile terminals, students will be able to carry visual communication with the teachers and enable teachers to guide their learning. A third-generation mobile communication (3G) technology has greatly accelerated the convergence of mobile communications and the Internet, the combination of mobile communication and education, greatly enhance their learning efficiency. The rapid development of new technologies in the context, $3 \mathrm{G}$ communications technology will be widely applied to education. With the practice of in-depth and awareness-raising, mobile education will showcase its endless charm.

Automatic response to question

VI. Future Developments

Problems of learners received, the server should be in accordance with the method of matching keywords searchable database, if found a similar problem is to answer, it automatically returns the appropriate answer to the questioner.

\section{Browsing questions and answers}

Learners through the system should visit the other student's questions and corresponding answers, in order to achieve knowledge-sharing with each other to improve.

\section{Sending notification of teaching activities}

System should send notification to students when they go out to, so that students can be in touch with the educational institution at any time, replacing a lot of inconvenience.

\section{References}

[1] Yazhen Zhang, Jian Li, "Application of 3G-based mobile learning in teacher training”, 2011 Fourth International Conference on Information and Computing, p27-29

[2] Wu Junqi, Qi Lili, Zhengbing Hu, “3G Phone-based Mobile Learning for Improving K-12 Tearchers'Educational Technology in Rural Area", 2010 Second International Workshop on Education Technology and Computer Science, p821-825

[3] John Traxler, "Defining Mobile Learning”, IADIS International Conference Mobile Learning 2005, p261-266

[4] Tiffany A. Koszalka, G.S. Ntloedibe-Kuswani, "Literature on the safe and disruptive learning potential of mobile technologies", 2010 Open and Distance Education Vol. 31, No. 2, August 2010, 139-157

[5] Kohei Arai, Herman Tolle, " Efficiency Improvement of E-Learning Document Search Engine for Mobile Browser" , International Journal of Research and Reviews in Computer Science (IJRRCS) Vol. 2, No. 6, December 2011, p1287-1291

[6] Supyan Hussin, Mohd Radzi Manap, Zaini Amir \& Pramela Krish, "Mobile Learning Readiness among Malaysian Students at Higher Learning Institutes", Asian Social Science; Vol. 8, No. 12; 2012, p276-283

[7] Seppala \& H. Alamaki, "Mobile learning in teacher training” Journal of Computer Assisted Learning (2003) 19

[8] Keegan,Desmond, "The future of learning:from elearning to mlearning” Information Analyses. 2002.11,pp44-65 